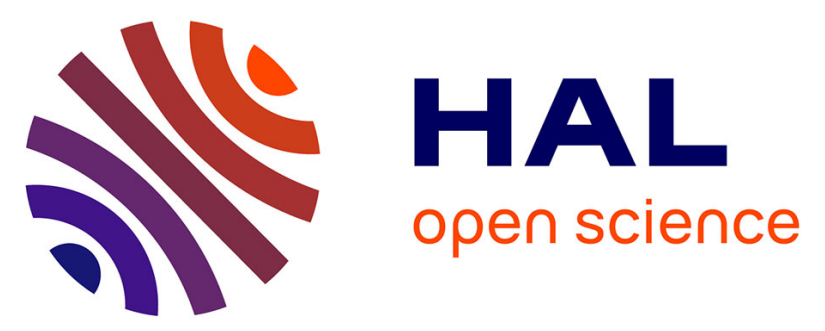

\title{
The Decay of Unstable Orientational States of Nematic Liquid Crystals Interacting with a Laser Field
}

\author{
G. Cipparrone, Dumitru Duca, C. Versace, C. Umeton, N. Tabiryan
}

\section{To cite this version:}

G. Cipparrone, Dumitru Duca, C. Versace, C. Umeton, N. Tabiryan. The Decay of Unstable Orientational States of Nematic Liquid Crystals Interacting with a Laser Field. Journal de Physique I, 1997, 7 (9), pp.1143-1153. 10.1051/jp1:1997113 . jpa-00247387

\section{HAL Id: jpa-00247387 https://hal.science/jpa-00247387}

Submitted on 1 Jan 1997

HAL is a multi-disciplinary open access archive for the deposit and dissemination of scientific research documents, whether they are published or not. The documents may come from teaching and research institutions in France or abroad, or from public or private research centers.
L'archive ouverte pluridisciplinaire HAL, est destinée au dépôt et à la diffusion de documents scientifiques de niveau recherche, publiés ou non, émanant des établissements d'enseignement et de recherche français ou étrangers, des laboratoires publics ou privés. 


\title{
The Decay of Unstable Orientational States of Nematic Liquid Crystals Interacting with a Laser Field
}

\author{
G. Cipparrone $\left({ }^{1}\right)$, D. Duca $\left({ }^{1}\right)$, C. Versace $\left({ }^{1}\right)$, C. Umeton $\left({ }^{1, *}\right)$ \\ and N.V. Tabiryan $\left({ }^{2}\right)$ \\ $\left({ }^{1}\right)$ Istituto Nazionale per la Fisica della Materia, Unità di Cosenza and Dipartimento di Fisica, \\ Università della Calabria, 87036 Rende (CS), Italy \\ $\left({ }^{2}\right)$ Center for Research and Education in Optics and Lasers (CREOL), \\ University of Central Florida, P.O. Box 162700, Orlando, FL 32816-2700, USA
}

(Received 15 July 1996, received in final form 21 May 1997, accepted 26 May 1997)

PACS.42.65.-k - Nonlinear optics
PACS.61.30.-v - Liquid crystals

\begin{abstract}
We show that the rate of relaxation is an important characteristic of the dynamics of critical systems undergoing second order phase transition, which are, in fact, still in effective motion even after the order parameter is reaching its steady state value. As a model system, we investigate the instability of the orientational state of a nematic liquid crystal acted on by a laser field and present the measurement of the initial fluctuations that start up the decay of the unstable state.
\end{abstract}

\section{Introduction}

Although criticality is a fundamental characteristic of many phenomena, only few of them allow for a straightforward experimentation. In this sense, the reorientation of a Nematic Liquid Crystal (NLC) under the influence of an external field represents an exceptional opportunity for investigating critical phenomena and their dynamics [1-3]. In particular, this is true for optically induced instabilities of the NLC-orientation. First of all, the use of laser beams allows to realize a spatially localized influence on the NLC; second, non-linear optical phenomena provide an accurate and extremely straightforward mean for registration of the NLC reorientation. The so called "self-heterodyning" technique [4] has been previously utilized, indeed, both for the study of light-NLC interaction and for the determination of material parameters of the NLC sample $[5,6]$.

The director reorientation observed in a NLC acted on by a laser field is known as "Optical Freedericksz Transition" (OFT) [7,8]. If the optical anisotropy of NLC (which is positive in most materials of interest) is small, the OFT is essentially similar to the Freedericksz transition induced by a quasistatic electric or magnetic fields [9]. Theoretical investigation of the dynamics of Freedericksz transition in a magnetic field, with account of thermal fluctuations, have been presented in several papers [3,10-13]. The dynamics of OFT has been discussed so far in

(*) Author for correspondence (e-mail: umeton@fis.unical.it) 


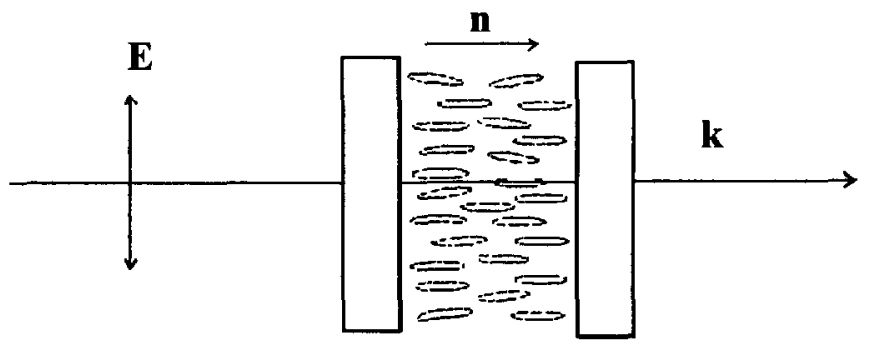

Fig. 1. - Experimental geometry of the NLC cell.

the deterministic limit, with the purpose of carrying out a linear stability analysis of spatially inhomogeneous orientational states that can be generated above the threshold intensity [14]. All these investigations of relaxation phenomena adopt the basic results of the theory of decay of unstable states [15-19]. According to this theory, two characteristic times are distinguished. One of them describes the relaxation of small fluctuations around a stationary stable state and is referred to as the "differential relaxation time". The second one represents the time interval which is required for the system to leave the immediate vicinity of an unstable state, and is called "Mean First Passage Time" (MFPT) [13-17].

In the present paper, we use OFT as a model system to investigate the dynamics of the second order phase transition. In particular:

1) We show that the rate (speed) of relaxation from the initial to the final equilibrium state of the system achieves its biggest value at an intermediate stage of the process, and the order parameter of the system is in effective motion even in the immediate vicinity of the final equilibrium state. We present the results of both theoretical and experimental investigations, showing that the characteristic time of effective motion (together with the differential relaxation time and the MFPT) is an important feature of the dynamics of critical phenomena. In addition, a new physical meaning of the MFPT is suggested that allows for an unambiguous and precise experimental characterization.

2) The initial fluctuations that start up the critical reorientation of the NLC are measured for the first time for OFT.

3) Our results provide evidence for a new phenomenon: non-critical enhancement of the startup fluctuations due, probably, to a strong interaction between additive and multiplicative noises.

\section{The Model System and the Main Equations}

As mentioned above, OFT in NLC's proves to be an attractive model for the study of critical phenomena and is highly suitable for experimentation, which can be simply realized by using a low power $\mathrm{cw}$ laser and exploiting the "self-heterodyning" technique $[4,5]$. Self phase modulation of the laser beam due to reorientation of the NLC director gives rise to the formation of a well known ring-shaped interference fringe pattern in the far field zone. The number of observed rings provides a direct measure of the NLC-reorientation angle and has been used, since many years, to characterize optically induced orientational phenomena in NLC [7,8]. Let us consider a homeotropically aligned NLC sample: molecules are perpendicular to the substrates of the cell and strongly anchored. The light beam is normally impinging and propagates along the $z$-axis, which is normal to the cell substrates (Fig. 1). The spot size in the beam 
waist is considered to be larger than (or, at least, comparable with) the thickness of the NLC layer. Then, a small area of the cell is being tested, thus ensuring smoothness of the profile of reorientation in the plane which is transverse to the propagation direction of the beam. Such a localization of the process appears impossible to be realized by means of quasistatic electric or magnetic fields since, in these cases, the dynamics of the NLC reorientation is influenced by the transient pattern formation and by the transverse modulation due to fluctuations of the NLC $[12-14,20,21]$. Thus, in our case, the critically enhanced mode of director fluctuations can be approximated by $\Theta(z, t)=\theta(t) \sin (\pi z / L)$ where $\Theta(z, t)$ is the reorientation angle as a function of the $z$-coordinate and time $t$, while $\theta(t)$ is the biggest reorientation angle that is achieved, as a function of time, at the centre of the NLC-cell with hard anchoring at the boundary layers (at $z=0$ and $z=L$ ). The equation for the dynamics of $\theta(t)$ can be presented in the form [7]

$$
\frac{\partial \theta}{\partial t^{\prime}}=(\rho-1) \theta-\mu \theta^{3}
$$

where $t^{\prime}=t / \tau$ is the time in units of the characteristic relaxation time

$$
\tau=\frac{\gamma L^{2}}{\pi^{2} K_{3}}
$$

and

$$
\rho=\frac{I}{I_{\mathrm{F}}}
$$

is the light intensity in units of the characteristic threshold value of the OFT:

$$
I_{\mathrm{F}}=\left(\frac{\pi}{L}\right)^{2} \frac{c \varepsilon_{\|} K_{3}}{\varepsilon_{\mathrm{a}} \sqrt{\varepsilon_{\perp}}}
$$

In equation (1) we have taken

$$
\mu=\frac{1}{4}\left(\left(1-\frac{9 \varepsilon_{\mathrm{a}}}{4 \varepsilon_{\|}}\right) \rho-\frac{K_{3}-K_{1}}{K_{3}}\right)
$$

and the conventional notations are used for the light speed in vacuum $(c)$, NLC orientational viscosity $(\gamma)$, elastic constants $\left(K_{\imath}\right)$, dielectric anisotropy $\left(\varepsilon_{\mathrm{a}}=\varepsilon_{\|}-\varepsilon_{\perp}\right)$ at the optical frequency $(\omega)$ and cell thickness $(L)$. We will assume $\mu>0$ which is the case for most of NLC's. In deriving equation (1), the lowest order non-linear term in $\theta^{3}$ has been retained in order to account for the stabilization of the director reorientation over the threshold. Furthermore, in equation (1) we have not included the stochastic force since it plays a significant role only near the critical states, (particularly in the initial stage of the decay of the unstable orientational state) while in the intermediate regime the dynamics is mainly determined by the nonlinearity of the system $[3,15,19]$. In the process of interaction with an electromagnetic radiation this dynamics reveals itself through the non-linear phase shift $\Phi$ induced in the laser light when crossing the NLC-cell. In fact, due to the large optical anisotropy of the NLC (typically, $\varepsilon_{\mathrm{a}} \approx$ $0.5)$ and the very large value of the wavenumber at the optical frequency $\left(\omega / c \approx 10^{5} \mathrm{~cm}^{-1}\right)$, even a small reorientation of the NLC director results in a remarkable phase shift [7]:

$$
\Phi=\frac{\omega \varepsilon_{\mathrm{a}} L}{4 c \sqrt{\varepsilon_{\perp}}} \theta^{2} .
$$

From the experimental point of view, we can register the squared reorientation angle by measuring $\Phi$. Rewriting equation (1) in terms of $\Phi$, we get indeed:

$$
\frac{\mathrm{d} \Phi}{\mathrm{d} t}=2 \Gamma \Phi(t)\left[1-\frac{\Phi(t)}{\Phi_{\infty}}\right]
$$


where

$$
\Gamma=\frac{1}{\tau}(\rho-1) \text { and } \Phi_{\infty}=(\rho-1) \frac{\omega \varepsilon_{\mathrm{a}} L}{4 c \mu \sqrt{\varepsilon_{\perp}}} .
$$

$\Phi_{\infty}$ represents the steady state limit reached by $\Phi(t)$ for a given value of $\rho$. From equation (6) we have therefore:

$$
V(t)=\frac{\mathrm{d} \theta^{2}}{\mathrm{~d} t}=\frac{(\rho-1)}{\mu \Phi_{\infty}} \frac{\mathrm{d} \Phi}{\mathrm{d} t}=2 \frac{(\rho-1)^{2}}{\tau \mu \Phi_{\infty}} \Phi(t)\left[1-\frac{\Phi(t)}{\Phi_{\infty}}\right] .
$$

\section{The Rate of Relaxation}

The physical quantity we are aimed to study is $V(t)$, the "rate" of decay of the squared value of the director reorientation angle. Measurement of this quantity has not only fundamental but also practical interest: Reorientation of NLC induced by laser beams is demonstrated to provide unique opportunities for the characterization of energetic parameters of the beams; by that, the measurement of the rate of relaxation allows to carry out measurements in periods which are one order of magnitude smaller than the characteristic relaxation time [22].

First of all, we note that $V(t)$ is small both at the beginning and at the late stages of the process and reaches its maximum value $V_{\mathrm{m}}$ when the phase shift becomes half of its steady state value (i.e. $\left.\Phi(t)=\Phi_{\infty} / 2\right)$ :

$$
V_{\mathrm{m}}=\frac{(\rho-1)^{2}}{2 \tau \mu}
$$

Proceeding from the solution of equation (7)

$$
\Phi(t)=\frac{\Phi_{\infty}}{1+\frac{\Phi_{\infty}-\Phi_{0}}{\Phi_{0}} \mathrm{e}^{-2 \Gamma t}}
$$

(where $\Phi(t=0)=\Phi_{0}$ ) we obtain that $V_{\mathrm{m}}$ is reached at the time instant

$$
t_{\mathrm{m}}=\frac{1}{2 \Gamma} \ln \left(\frac{\Phi_{\infty}-\Phi_{0}}{\Phi_{0}}\right) \approx \frac{1}{2 \Gamma} \ln \left(\frac{\Phi_{\infty}}{\Phi_{0}}\right)=\frac{1}{2 \Gamma} \ln \left(\frac{\theta_{\mathrm{m}}^{2}}{\theta_{0}^{2}}\right)
$$

where $\theta_{0}$ and $\theta_{\mathrm{m}}$ indicate the initial and the maximum reorientation angles, respectively. Thus, we can introduce a quantity, $t_{\mathrm{m}}$, which is of the order of magnitude of the MFPT but has a new and well defined physical meaning: it is the time when the rate of escape of the squared director orientation angle from its unstable equilibrium value is maximum. We underline that the definition of $t_{\mathrm{m}}$ does not require any arbitrary choice of particular conditions and allows for an unambiguous and precise experimental characterization.

An interesting physical consequence of our considerations concerns the concept of the achievement of the steady state. In fact, the condition that the director orientation has achieved the $1 / e$ level of its steady state value does not imply achievement of equilibrium. The system can be regarded as stationary only if the speed of relaxation also becomes sufficiently small. The time $t_{\mathrm{v}}$ needed for $V(t)$ to reach the $1 / e$ level of its maximum can be easily calculated and is:

$$
t_{\mathrm{v}}=t_{\mathrm{m}}+\frac{1}{2 \Gamma} \ln \left(\frac{1+\sqrt{1-\mathrm{e}^{-1}}}{1-\sqrt{1-\mathrm{e}^{-1}}}\right) \approx t_{\mathrm{m}}+\frac{1.1}{\Gamma} .
$$

This time interval is larger than $t_{\Phi}$, that is the relaxation time needed for $\theta^{2}$ to reach the $1 / e$ level of its maximum:

$$
t_{\Phi}=t_{\mathrm{m}}+\frac{1}{2 \Gamma} \ln (e-1) \approx t_{\mathrm{m}}+\frac{0.3}{\Gamma}
$$




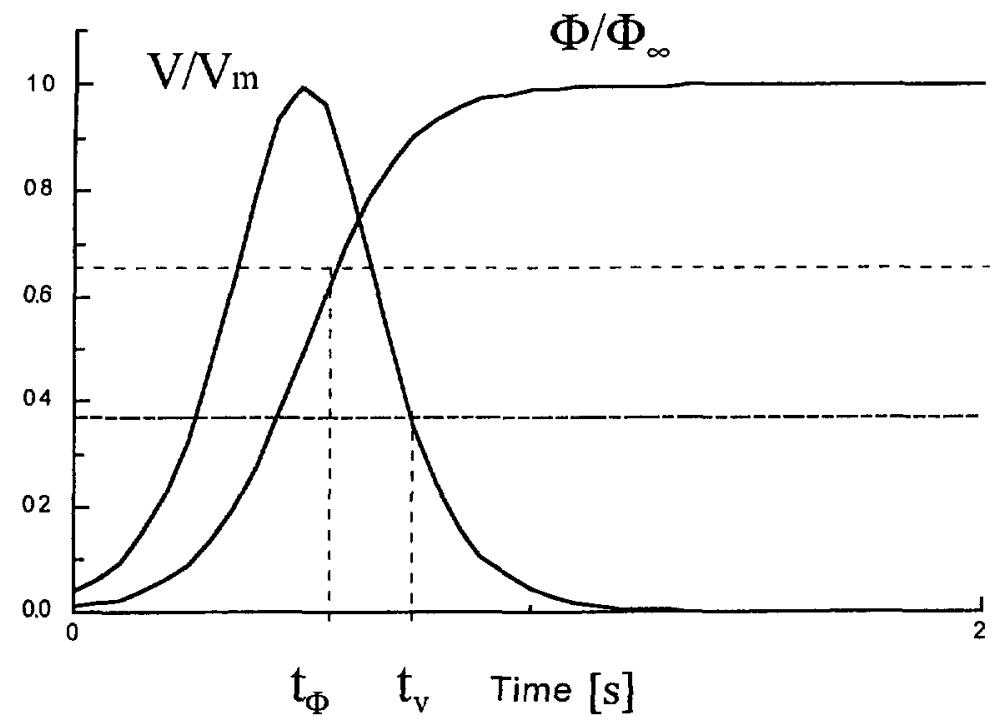

Fig. 2. - Typical time behaviour of $\Phi(t) / \Phi_{\infty}$ and $V(t) / V_{\mathrm{m}}$ as calculated from equations $(11,7)$.

Figure 2 shows this difference for a particular value of $\left(\Phi_{\infty}-\Phi_{0}\right) / \Phi_{0}$ : owing to the dependence of $\Gamma$ on $\rho$ given by equation (8), in the proximity of the critical point $\left(I \approx I_{\mathrm{F}}\right)$ the difference $t_{\mathrm{v}}-t_{\Phi} \approx 0.8 / \Gamma$ can become appreciable; we stress out that this peculiarity of the phenomenon is due to its non-exponential relaxation behaviour.

It is also interesting to find out the time interval during which the system is in motion. This can be calculated as the full width at half maximum of the lineshape of $V(t)$ :

$$
\Delta t=\frac{1}{\Gamma} \ln \left(\frac{1+\sqrt{\frac{1}{2}}}{1-\sqrt{\frac{1}{2}}}\right) \approx \frac{1.7 \tau}{\rho-1} .
$$

$\Delta t$ is determined only by the excess of the control parameter over the critical threshold value $I_{\mathrm{F}}$ and by the time constant $\tau$ which is a characteristic of the medium. We note that the stochastic nature of the phenomenon determines the relaxation time of the director and its rate, but does not influence the effective motion time $\Delta t$.

\section{Experimental Set-Up and the Method}

The light source is an $\mathrm{Ar}^{+}$laser (Innova 90 by Coherent) operating at the wavelength $\lambda=514.5 \mathrm{~nm}$. After a shutter and a calibrated system of beam splitter and power meter, the laser light is linearly polarized and focused $(f=150 \mathrm{~mm}$ ) on the cell (thickness $L=50 \mu \mathrm{m}$ ). The NLC is the E7 (by British Drug Houses, parameter values $\varepsilon_{\|}=3$, $\varepsilon_{\perp}=2.4, K_{3}=1.2 \times 10^{-6}$ dyne, $\gamma=1.2$ Poise), homeotropically aligned and thermostabilized at $24^{\circ} \mathrm{C}$. The beam has a Gaussian profile of intensity with a spot size in the waist: $w_{0}\left(\mathrm{HWe}^{-1} \mathrm{M}\right)=35 \mu \mathrm{m}$. The outgoing beam is chopped and its central part is detected by a photodiode placed behind a $200 \mu \mathrm{m}$ pinhole. The detected signal is sent to a lock-in amplifier (model 5208 by EG\&G) which improves the signal to noise ratio, and then to the data 


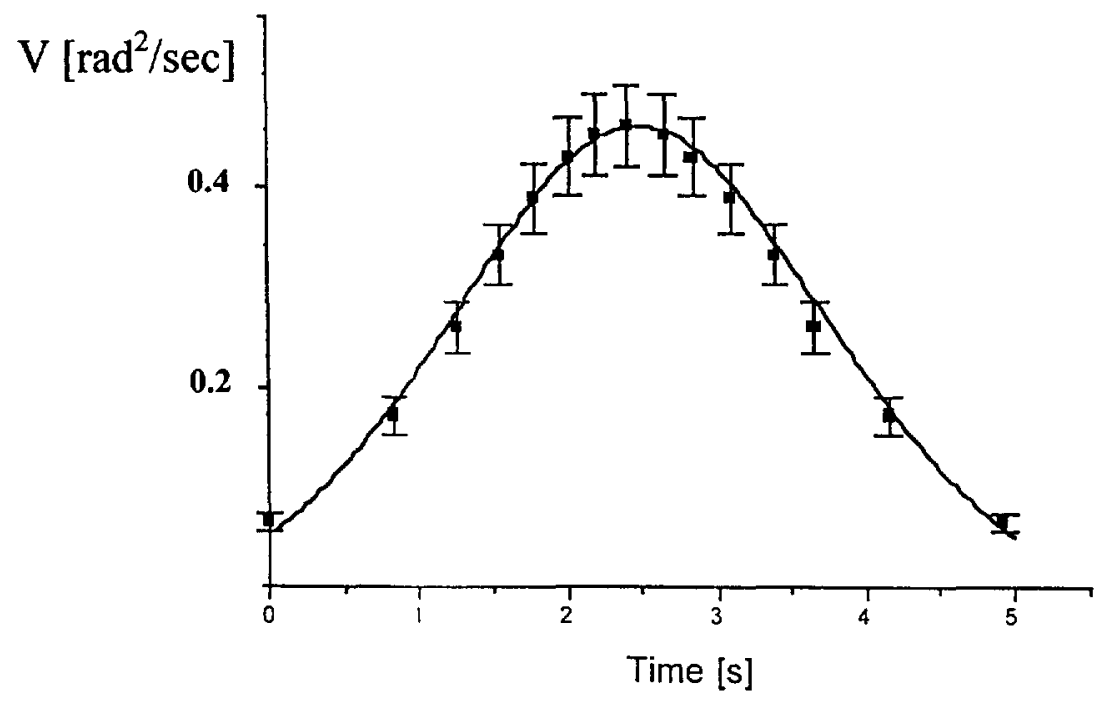

Fig. 3. - Typical experimental time behaviour of $V(t)$ as determined by following the dynamics of the ring pattern formation. In this case, $\lambda=514.5 \mathrm{~nm}$ and $P / P_{\mathrm{F}}=2.14 \pm 0.07$. acquisition system (Data 6000 by Data Precision) triggered by the shutter. The phase shift is measured by registering the number of circular interference fringes at the output of the NLC:

$$
\Phi(t)=2 \pi N(t)
$$

and the rate of relaxation $V(t)$ is measured by registering the frequency of oscillations in the intensity on the axis of the beam. The detailed discussion of this so called "self-heterodyning" method is presented elsewhere [4].

\section{Measurements}

5.1. The Rate of Relaxation. - The threshold power of the OFT has been found to be $P_{\mathrm{F}}=(188 \pm 6) \mathrm{mW}$ corresponding to the threshold power density $I_{\mathrm{F}}=4.9 \mathrm{~kW} / \mathrm{cm}^{2}$, a value which is slightly higher than the theoretical one $\left(4.6 \mathrm{~kW} / \mathrm{cm}^{2}\right)$ as calculated by means of equation (4). This circumstance evidences that the plane wave approximation made in deriving equation (1) is well valid.

Experiments for the determination of the rate of relaxation have been carried out at power values up to $\rho=P / P_{\mathrm{F}} \sim 2.14$. The particular experimental results presented in Figure 3 can be perfectly fit with the aid of analytical expressions $(9,11)$ and parameter values $\Phi_{\infty}=80$, $\Phi_{0}=4.5, \Gamma=0.567 \mathrm{~s}^{-1}$ The value of $\Gamma$ is in agreement with the theoretical evaluation $\Gamma=0.45 \mathrm{~s}^{-1}$ given by expression (8), but the value $\Phi_{\infty}=80$ corresponds to a magnitude of the steady state reorientation of the NLC of the order of $1 \mathrm{rad}(\mathrm{Eq}$. (6)). Thus, the obtained good fit is, in fact, quite unexpected and our physical system is an example of a system where the analytical expressions obtained at the limit of some parameter value $x \ll 1$, work fairly well up to $x \sim 1$.

Experimental values of $V_{\mathrm{m}}$ as a function of $\rho$ have been obtained by the plots of $V(t)$ (curves of the kind of Fig. 3) detected by means of the "self-heterodyning" method at different values of the impinging power. Far from the critical region $\left(P \gg P_{\mathrm{F}}\right)$, there is a saturation in $\Phi_{\infty}$. 


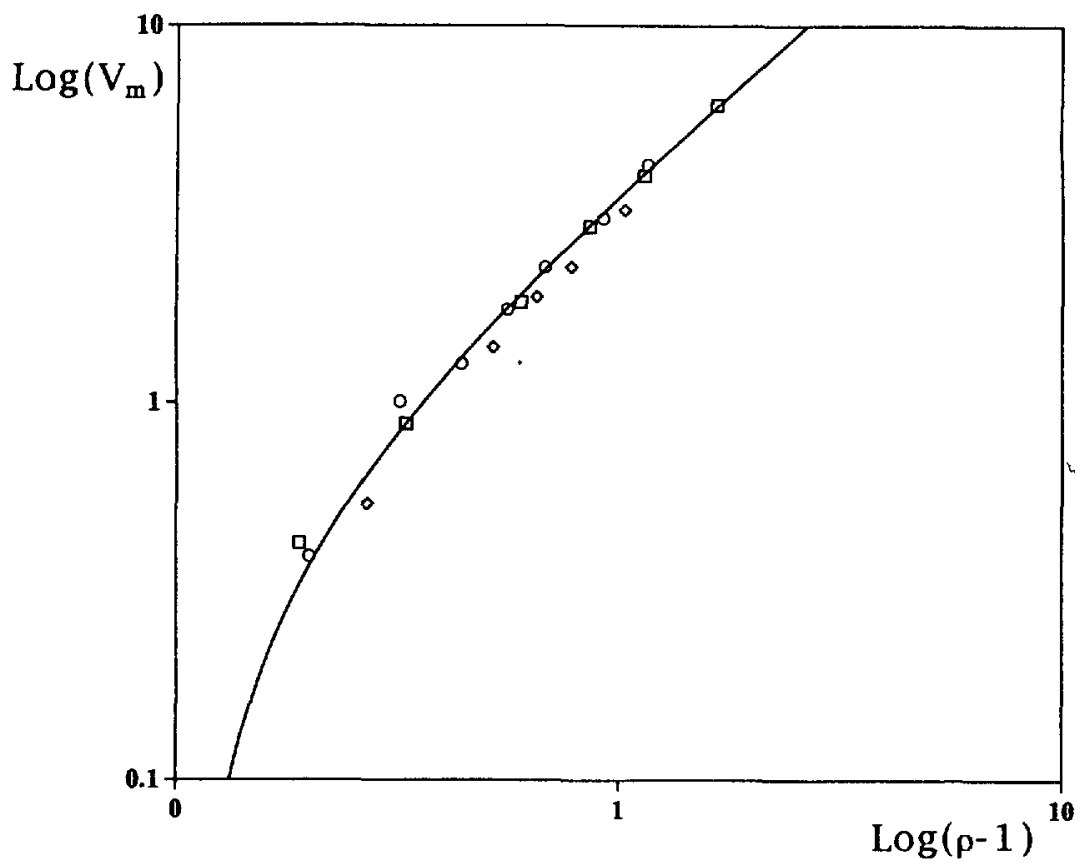

Fig. 4. - Experimental values of $\log \left(V_{\mathrm{m}}\right)$ versus $\log (\rho-1)$ for all the used wavelengths. Triangles refer to $\lambda_{1}=488 \mathrm{~nm}$, circles to $\lambda_{2}=496 \mathrm{~nm}$ and squares to $\lambda_{3}=514.5 \mathrm{~nm}$.

Indeed, following equations $(5,8,9)$, for $I \gg I_{\mathrm{F}}, \Phi$ becomes almost independent of $\rho$ and reaches the value $\Phi_{\text {csat }}$. Therefore, the intensity dependence of the maximum relaxation rate becomes linear:

$$
V_{\mathrm{m}}=\left(\Phi_{\infty \mathrm{sat}} / 2 \tau\right)(\rho-1)
$$

This kind of "saturation" is evident in Figure 4, where $\log \left(V_{\mathrm{m}}\right)$ versus $\log (\rho-1)$ is not a straight line with a fixed angular coefficient.

Finally, the plot of $1 / \Delta t$ (the inverse of the linewidth of $V(t)$ ) is presented versus $\rho-1$ in Figure 5: the linear dependence predicted for $1 / \Delta t$ by equation (15) is clearly shown.

5.2. Start-UP Fluctuations. - Stochastic fluctuations play the fundamental role of initiating the transition from the unstable to the stable equilibrium states. However, because of experimental difficulties, these fluctuations were recently characterized only for the case of the quasistatic electric field induced Freedericksz transition in NLC [21]. It has been found that: a) the effect of misalignment of the boundary layers of NLC is negligible compared to the effect of fluctuations; b) the magnitude of fluctuations and their dependence on the vicinity to the critical region is well described by the conventional theory of thermal fluctuations of the NLC-orientation [9]; c) the reorientation is accompanied by transverse patterns because of the large sizes of the electrodes (needed to apply the electric field) compared with the thickness of the NLC-layer.

In a previous paper [5], we have underlined the opportunity, given by the OFT, to carry out characterization of the start-up fluctuations for critical systems. Indeed, the relaxation process can be described in its initial stage by a linearization of equation (11):

$$
\ln \Phi(t)=\ln \Phi_{0}+2 \Gamma t .
$$




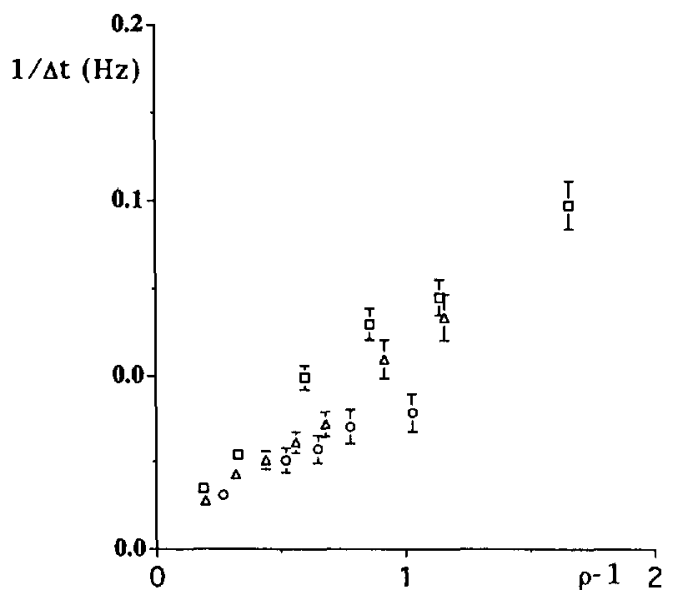

Fig. 5. - Trend of $\Delta t$ versus $\rho-1$ for all the used wavelengths. Triangles refer to $\lambda_{1}=488 \mathrm{~nm}$, circles to $\lambda_{2}=496 \mathrm{~nm}$ and squares to $\lambda_{3}=514.5 \mathrm{~nm}$.

With this approximation, the plot of $\ln \Phi(t)$ versus time is a straight line whose extrapolation to the time instant $t=0$ gives $\ln \Phi_{0}$ and, thus, by means of equation (6), it allows for the determination of the initial squared fluctuation angle $\theta_{0}^{2}$. By that, due to the possibility to limit the size of the beam, one can expect that the reorientation takes place smoothly across the beam, without generation of transverse instabilities that would affect the dynamics of the process. Measurements have been repeated up to 15 times for a given value of $\rho$, with the aim of checking whether the initial fluctuation state is the same for all the experiments which are carried out in the same conditions or it changes when the experiment is repeated. Furthermore, in order to investigate the dependence of $\left\langle\theta_{0}^{2}\right\rangle$ on the proximity to the critical intensity, determinations of $\Phi_{0}$ have been also performed at different values of $\rho$.

In Figure 6, the plot of the experimental values of $\ln \Phi(t)$ versus time shows a satisfactory agreement with the approximate linear dependence given by equation (18). The dependence of the average $\left\langle\theta_{0}^{2}\right\rangle$ on $(\rho-1)^{-1}$ obtained from the experimental determination of $\ln \Phi_{0}$ is shown in Figure 7. The large variance ( $\sigma^{2}$ is of the order of $\left.40 \%\right)$, is not due to experimental errors, which always remain of the order of $8 \%$, but reflects the stochastic origin of $\Phi_{0}$ and indicates that, each time the experiment is repeated under the same conditions, the reorientation of the NLC does not start from some average level of fluctuations, but basically it may be a drag of the initial state of orientation encountered at the moment the light beam impinges on the sample. However, the enhancement of the initial fluctuations in the critical region of light intensity (Fig. 7) indicates that the reorientation process is proceeded by an enhancement of fluctuations.

\section{Discussion}

Registered values of $\left\langle\theta_{0}^{2}\right\rangle$ turn out to be remarkably higher than that estimated by the simple expression [9-12]:

$$
\left\langle\theta_{0}^{2}\right\rangle=\frac{k_{\mathrm{B}} T}{L K_{3}} \approx 10^{-4}
$$




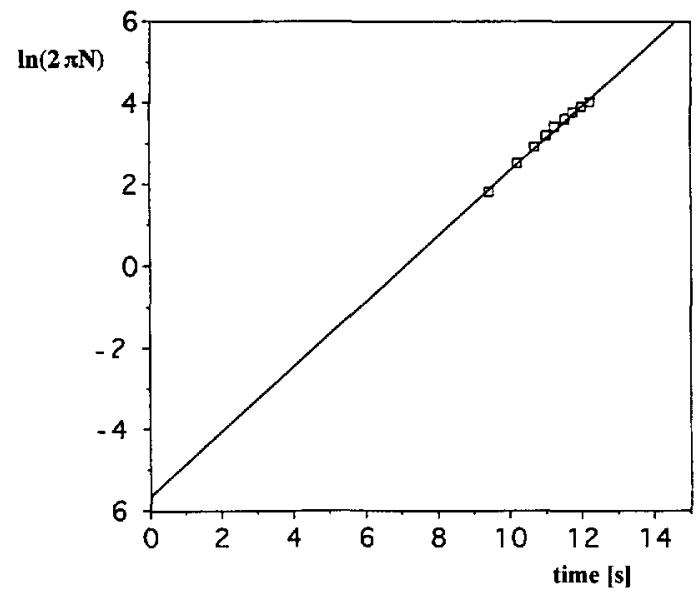

Fig. 6. - Initial trend of $\ln [2 \pi N(t)]$ versus time; data refer to one of the 14 experiments performed at $\rho=1.86$.

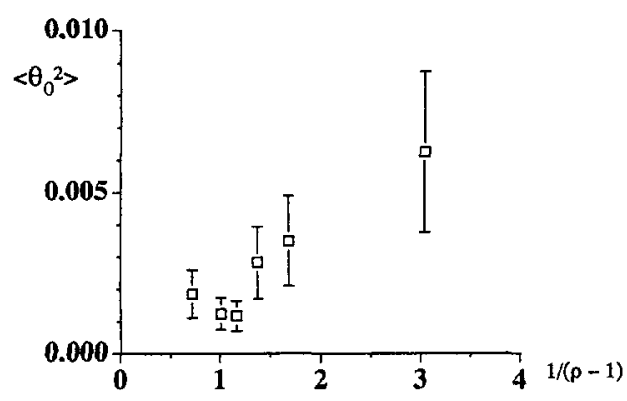

Fig. 7. - Dependence of average $\theta_{0}^{2}$ on $(\rho-1)^{-1}$. The bar does not indicate experimental errors but the statistical $\sigma$ value obtained from different measurements performed in the same experimental conditions.

In order to explain this observation, we have performed an analysis of the main possible reasons:

a) Pretilt or non-ideal alignment of NLC at the cell boundaries.

These contributions, though important in many situations, would be either independent on the laser beam intensity or, would increase with it due to non-threshold effects of light-induced reorientation: this is not our case. Moreover, the effects of pretilt has been found to be negligible in [21].

b) Non-ideal normal incidence of the beam on the NLC-cell.

As for the non-ideal alignment, one would expect that the measured level of initial orientation should increase due to non-threshold reorientation of NLC in the obliquely impinging light beam. It is easy to evaluate that the role of the finite angle made by the director and light beam due to focusing is negligible in our experimental situation (beam focused by a lens of $f=15 \mathrm{~cm}$ focal length and waist radius of the beam $=35 \mu \mathrm{m}$ ).

c) Variation of material parameters.

In experiments in which use is made of laser beams, heating of the material due to absorption of laser radiation can lead to variation of the material parameters such as viscosity, elastic 
constants and optical anisotropy. For the NLC used in our experiment, the absorption constant $\alpha$ is of the order of $10^{-2} \mathrm{~cm}^{-1}$ Thus, only a negligible part of radiation is being absorbed in the layer of thickness $L=50 \mu \mathrm{m}$. Thermal effects have therefore a negligible contribution in our results.

d) Noise introduced by the scattering of light.

Laser-induced critical phenomena are peculiar stochastic processes where the intrinsic fluctuations (additive noise) result in a strong light scattering and, hence, in strong fluctuations of external parameter (multiplicative noise). We note that, with a scattering cross-section of NLC of the order of $\sigma=5 \mathrm{~cm}^{-1}$ (for the ordinary wave), and a thickness of the NLC-layer $L=50 \mu \mathrm{m}$, the intensity of the scattered light $\left(\rho_{\mathrm{s}}=\sigma L \rho=2.5 \times 10^{-2} \rho\right)$ can reach considerable values even when the intensity of the incident beam is of the order of the OFT threshold $(\rho=1)$. Furthermore, if we consider that the scattering is many orders of magnitudes larger than in isotropic liquids for a large region of incidence angles (up to $1 \mathrm{rad}$ ) [23], the reorientation of the NLC-director due to the scattered light can reach values $\theta \sim 10^{-2}$, very close to the observed ones. We also note that in the critical region of second order phase transition, both the fluctuations and the light scattering critically enhance. As seen in Figure 7, we actually observe this critical behaviour near the OFT threshold. For large intensities, far from the critical region, the character of the dependence of the start-up fluctuations on the light intensity apparently changes and the level of fluctuations shows the tendency to increase with increasing intensity. Though our data are not sufficient for final conclusions, this increase of $\left\langle\theta_{0}^{2}\right\rangle$ with intensity can be treated in terms of the mentioned effect of multiplicative scattering noises; indeed, away from the critical region, this effect may be dominant. We think therefore that both mentioned phenomena (fluctuations of laser intensity and the multiplicative noise induced by the additive-one) should be further investigated to describe the measured values of the mean level of start-up fluctuations and their dependence on the proximity to the critical point.

In conclusion, we have characterized the relaxation and stochastic properties of the unstable orientational states of a NLC acted on by a laser beam. The rate of relaxation has a maximum at an intermediate stage of the development of the instability and, depending on the proximity to the critical point, it can be still large even when the relaxing parameter is considered to have reached its steady state. The time of "effective motion" is determined by the characteristic time constant of the medium and by the proximity to the critical point. Finally, we have measured the director fluctuations which initiate the second order phase transition. The amplitudes of these fluctuations are very much at variance for different measurements performed in the same experimental conditions. Their mean square value decreases with increasing light intensity, staying, however, considerably higher than that expected in the framework of the conventional thermodynamics.

\section{Acknowledgments}

This work is partially supported by M.U.R.S.T., Italy. Researches of N.V. Tabiryan have been partially supported by A. von Humboldt Foundation, Germany. One of the authors (N.T.) acknowledges encouraging discussions of the problem with F. Sague's. 


\section{References}

[1] Horsthemke W. and Lefever R., Noise induced transitions (Springer, Berlin, 1984).

[2] Horsthemke W., Doering C.R., Lefever R. and Chi A.S., Phys. Rev. A 31 (1985) 1123.

[3] Sague's F. and San Miguel M., Phys. Rev. A 32 (1985) 1843.

[4] Cipparrone G., Duca D., Umeton C., Versace C. and Tabiryan N.V., Mol. Cryst. Liq. Cryst. 261 (1995) 187.

[5] Cipparrone G., Duca D., Umeton C. and Tabiryan N.V., Phys. Rev. Lett. 71 (1993) 3955.

[6] Cipparrone G., Duca D., Versacce C., Umeton C. and Tabiryan N.V., Mol. Cryst. Liquid Cryst. 266 (1995) 263.

[7] Tabiryan N.V., Sukhov A.V. and Zel'dovich B.Ya., Mol. Cryst. Liquid Cryst. 136 (1986) 1 , and references quoted therein.

[8] Khoo I.C. and Wu S., Optics and nonlinear optics of liquid crystals (World Scientific, 1993), and references quoted therein.

[9] de Gennes P., The physics of liquid crystals (Clarendon Press, Oxford, 1974).

[10] Brochard F., Pieranski P. and Guion E., Phys. Rev. Lett. 28 (1972) 1681.

[11] Pieranski P., Brochard F. and Guion E., J. Phys. France 34 (1973) 35.

[12] Sague's F. and San Miguel M., Phys. Rev. A 33 (1986) 2769.

[13] San Miguel M. and Sague's F., Phys. Rev. A 36 (1987) 1883.

[14] Marquis F., Meystre P., Wright E.M. and Kaplan A.E., Phys. Rev. A 36 (1987) 875.

[15] Arecchi F.T. and Degiorgio V., Phys. Rev. A 3 (1971) 1108.

[16] Arecchi F.T. and Politi A., Phys. Rev. Lett. 45 (1980) 1219.

[17] Haake F. and Haus J.W., Phys. Rev. A 23 (1981) 3255.

[18] Suzuki M., Phys. Lett. A 67 (1978) 339.

[19] de Pasquale F. and Tombesi P., Phys. Lett. A 72 (1979) 7.

[20] Grigutsch M., Kloepper N., Schmiedel H. and Stannarius R., Mol. Cryst. Liquid Cryst. 261 (1995) 283.

[21] Kuehnau U., Schmiedel H. and Stannarius R., Mol. Cryst. Liquid Cryst. 261 (1995) 293.

[22] Murazyan A.L., Tabiryan N.V., Vogeler T. and Tschudi T., Optik: Int. J. Light and Electron Optics 101 (1996) 145.

[23] Val'kov A.Yu., Zubkov L.A., Kovshik A.P. and Romanov V.P., Sov. Phys. JETP Lett. 40 (1984) 1064. 